\title{
Thomas E. A. DALE, Pygmalion's Power: Romanesque Sculpture, the Senses, and Religious Experience
}

Éric Palazzo

\section{OpenEdition}

\section{Journals}

Édition électronique

URL : https://journals.openedition.org/ccm/7378

DOI : $10.4000 / \mathrm{ccm} .7378$

ISSN : 2119-1026

\section{Éditeur}

Centre d'études supérieures de civilisation médiévale/Université de Poitiers

\section{Édition imprimée}

Date de publication : 1 juin 2021

Pagination : 153-155

ISBN : 978-2-490783-09-0

ISSN : 0007-9731

Référence électronique

Éric Palazzo, «Thomas E. A. DALE, Pygmalion's Power: Romanesque Sculpture, the Senses, and Religious Experience », Cahiers de civilisation médiévale [En ligne], 254 | 2021, mis en ligne le 01 juin 2021, consulté le 02 décembre 2022. URL : http://journals.openedition.org/ccm/7378 ; DOI : https://doi.org/ $10.4000 / \mathrm{ccm} .7378$

\section{cc) (i) (2)}

Creative Commons - Attribution - Pas d'Utilisation Commerciale - Pas de Modification 4.0 International - CC BY-NC-ND 4.0

https://creativecommons.org/licenses/by-nc-nd/4.0/ 
Thomas E. A. Dale, Pygmalion's Power: Romanesque Sculpture, the Senses, and Religious Experience, University Park, The Pennsylvana State University Press, 2019.

On a pris l'habitude, à tort, de considérer la sculpture romane principalement à partir des grands ensembles en pierre qui s'imposent au regard à l'entrée des églises, qui se déploient dans l'espace intérieur des édifices ou bien encore le long des galeries des cloîtres. L'un des mérites de la monographie consacrée par Thomas Dale à la dimension sensorielle et à l'expérience religieuse de la sculpture romane réside précisément dans une conception élargie de ce matériau. Dans ce livre, l'a. s'intéresse autant à la sculpture sur pierre des grands portails romans ou des chapiteaux sculptés dans les cloîtres qu'à la production des reliquaires, des crucifix ou bien encore des vierges trônantes. Le titre du livre est clairement une allusion, comme s'en explique l'a. dans son introduction, au fantasme de la statue vivante et suggère que l'approche sensorielle de la sculpture romane s'inscrit dans le cadre de la création vivante qui est celui du mythe de Pygmalion. Or, il n'en est rien puisque l'animation sensorielle des sculptures médiévales permettant de les « rendre vivantes » repose sur des notions théologiques d'un tout autre ordre que celui suggéré par le mythe de Pygmalion. Malgré cela, on ne peut que souscrire à la thèse de $\mathrm{T}$. Dale selon laquelle l'animation de la sculpture romane, sous toutes ses formes artistiques, est fondamentalement basée sur la perception sensorielle des œuvres dans le but de générer l'animation des « images » ou des « figures » à des fins théologiques diverses.

Dans le premier chapitre, T. Dale se penche sur les statues « vivantes » du crucifix et de la Vierge trônante ou du Trône de Sagesse. S'appuyant sur une bibliographie fournie et à jour, l'a. procède à une sorte de synthèse sur ce thème de l'animation des sculptures en bois notamment, mais pas seulement, sous l'action de la force des sens provoquée par le spectateur ou, pour être plus précis, par le dévot qui procédait à ses pratiques de dévotion privée à partir de crucifix ou de statues dont certaines étaient aussi des reliquaires. Dans ces pages, l'a. remonte, à juste titre, à l'époque carolingienne où l'on trouve les traces - toujours controversées pour certaines de l'animation sensorielle des sculptures à partir de la pratique dévotionnelle. De telle sorte que T. Dale suggère implicitement l'existence d'une continuité entre ce processus d'animation sensorielle dans le haut Moyen Âge et ses prolongements dans la sculpture romane monumentale. Pour ma part, je ne peux que souscrire à cette idée comme j'ai essayé de le démontrer dans plusieurs publications. Dans ce même chapitre, on ne peut que suivre T. Dale dans la reprise qu'il fait à son compte des données relatives à l'animation «miraculeuse » de la statue de Sainte-Foy de Conques et que d'autres avant lui ont amplement développées.

Dans le chapitre suivant, l'a. revient sur le thème de la nudité dans la sculpture romane qu'il avait abordé ailleurs dans des articles. Le postulat défendu est simple : la nudité de certaines figures dans la sculpture monumentale romane s'appuie sur l'idée selon laquelle le corps nu n'est pas obligatoirement associé, en perspective chrétienne, au thème du péché et de la punition du corps. L'un des textes bibliques essentiels pour la démonstration est le passage de la Genèse où il est question de l'Homme créé à la ressemblance de Dieu (Genèse I, 26-27) qui a été l'objet d'une longue tradition exégétique étudiée notamment par Gilbert Dahan auquel l'a. ne fait pas référence. En même temps, T. Dale montre bien l'ambivalence existante entre ce que l'on pourrait qualifier de «nudité positive », qui renvoie au thème de la Création et à la valeur spirituelle du corps de l'Homme, et la «nudité négative » associée à l'expression du vice et du péché et dont témoignent certains grands ensembles sculptés de l'époque romane, tels que le portail occidental de la cathédrale d'Autun avec la représentation du jugement dernier que T. Dale décrit à nouveaux frais de façon convaincante.

Le chapitre trois s'appuie lui aussi sur des publications antérieures de l'a. et concerne cette fois l'important thème du portrait sculpté. T. Dale fait partie de ceux qui ont récemment permis de réviser la notion de portrait pour la période romane sur la base d'observations nouvelles faites à propos de portraits sculptés et de l'insistance sur certains organes de la tête localisés sur celle-ci, notamment les yeux dont le caractère globuleux relevé dans plusieurs effigies laisse penser à la volonté de l'artiste de « représenter » une sorte de projection du visuel dans le monde réel. Dans ce cadre, ce sont les portraits-reliquaires et les effigies funéraires qui font l'objet d'analyses poussées et de comparaisons avec des portraits figurant sur 
les sceaux. Sans surprise, T. Dale argumente en faveur du thème de la « Présence réelle» dans les portraits sculptés, soulignant par là l'importance accordée à cette époque à la découverte de l'individu en parallèle de l'affirmation de la présence du corps dans la conception médiévale de l'Homme. À un autre niveau, et à une autre époque, des commentaires similaires pourraient être faits à partir du développement, dans le cercle papal, de la prise en compte du corps individuel du pape à côté de l'existence de son corps institutionnel. Mais, dans ce chapitre, la dimension sensorielle est certes bien présente, mais ne constitue pas à mes yeux l'élément déterminant dans la mise en mouvement ou l'animation de ces portraits sculptés qui reposent plus, je pense, sur le thème de la prise en compte plus importante de la réalité corporelle à cette époque et ce, même si l'on suit l'a. au sujet de l'importance accordée à l'animation de ces sculptures à partir de certaines de leurs caractéristiques formelles, c'est-à-dire, « corporelles ».

Le chapitre quatre constitue, une fois encore, la reprise de nombreux éléments exposés ailleurs par l'a. Le thème central de ce chapitre est celui des difformités et des monstres dans l'imaginaire collectif médiéval et leurs traductions formelles dans la sculpture romane, en particulier dans l'art monumental. Dans ces pages d'une densité considérable, T. Dale fait preuve d'une grande maîtrise du sujet et de l'importante bibliographie qui lui a été consacrée par le passé. Les références à saint Augustin et à sa Cité de Dieu où l'on trouve de nombreuses observations sur les races fantastiques (monstrueuses parfois...) qui peuplent la terre constituent la solide base d'une réflexion théologique centrée sur la différence et la marginalité. Selon la logique défendue par saint Augustin comme par bon nombre de théologiens chrétiens après lui, ces races monstrueuses aux difformités physiques parfois horrifiantes seront sauvées par le christianisme et le message évangélique prôné par le Christ. À partir de là, T. Dale défend l'idée selon laquelle la dimension sensorielle a joué un rôle dans la perception physique et sensorielle par les « spectateurs » du Moyen Âge de la « réalité » de ces difformités et de ces monstruosités. Sur ce sujet, je dois avouer que je reste quelque peu dubitatif sur la solidité des démonstrations et des arguments avancés par l'a. Le sentiment qui se dégage est celui d'un renvoi, que l'on ressent artificiel, à la dimension sensorielle sur des thèmes tels que ceux de la difformité et de la monstruosité, certes bien présents sur certains ensembles sculptés mais pour lesquels je ne vois pas clairement l'animation sensorielle. Ces remarques portent principalement sur les importants passages consacrés par T. Dale à des ensembles qu'il connaît bien, tels que les chapiteaux et plaques sculptés des cloîtres de Saint-Michel-de-Cuxa et de l'abbaye de Silos. Tout ce qui est dit au sujet de ces deux monuments majeurs de l'art roman et de leurs sculptures est fort instructif et convaincant - notamment pour ce qui concerne la nécessité pour les moines d'apprendre à contrôler leurs émotions à partir de la confrontation avec les monstruosités représentées dans les cloîtres mais n'emporte pas vraiment l'adhésion du point de vue des implications sensorielles censées être au cœur de l'exploration du « pouvoir de Pygmalion ». À cela vient s'ajouter, une certaine incompréhension face aux exposés consacrés aux chapiteaux du chœur de l'église de Chauvigny, ou bien encore, au célèbre trumeau de Souillac et au non moins célèbre portail de Beaulieu-sur-Dordogne. Dans un cas comme dans l'autre, T. Dale ne semble pas avoir pris en considération les importantes différences de statuts de chaque édifice ainsi que l'espace dans lequel on trouve des «monstres » sculptés. Autrement dit, même si on ne peut être que d'accord avec l'a. lorsqu'il affirme que le processus de transformation de l'Homme peut passer par une utilisation négative ou positive des sens, ou bien que le portail roman apparaît tel un voyage spirituel proposé au fidèle à l'entrée de l'église, là encore, le discours sur la dimension sensorielle reste pour une large part à démontrer.

Des remarques analogues pourraient être faites au sujet du sixième et dernier chapitre qui constitue plus une sorte de synthèse générale sur la signification des théophanies romanes en lien avec certaines identités urbaines et plusieurs aspects essentiels de la théologie eschatologique qu'une approche renouvelée de ces portails à partir de leur animation sensorielle. Dans les pages dédiées au portail de la Pentecôte à Vézelay on ne peut que suivre l'a. dans l'observation générale au sujet de l'expérience religieuse de l'Esprit par les sens, mais, de mon point de vue, la démonstration n'est pas vraiment faite pour ce qui touche la dimension sensorielle de cette expérience.

Malgré ces quelques remarques critiques, le livre de $\mathrm{T}$. Dale va très certainement marquer un tournant dans l'historiographie de la sculpture romane par son ampleur de vue, par la diversité du matériau traité et 
par la manière dont l'a. a tenté, parfois avec succès, parfois moins, de souligner l'implication physique du spectateur médiéval face aux œuvres visuelles du christianisme de l'art roman et ce, même si la dimension sensorielle dont on perçoit intuitivement l'importance mériterait d'être dans certains cas mieux étayée.

Éric Palazzo UMR 7302 - CESCM / Université de Poitiers 